OPEN ACCESS

Edited by:

Cao Yong chang,

Sun Yat-sen University, China

Reviewed by:

Md. Golzar Hossain,

Bangladesh Agricultural University,

Bangladesh

Feifei Yin,

Hainan Medical University, China

*Correspondence:

Yujuan Niu

yujuanniu@163.com

Specialty section:

This article was submitted to

Virology,

a section of the journal

Frontiers in Microbiology

Received: 28 September 2021

Accepted: 20 December 2021

Published: 17 January 2022

Citation:

$\mathrm{Ma} \mathrm{H}$ and Niu Y (2022)

Metabolomic Profiling Reveals New Insight of Fowl Adenovirus Serotype 4

Infection.

Front. Microbiol. 12:784745.

doi: 10.3389/fmicb.2021.784745

\section{Metabolomic Profiling Reveals New Insight of Fowl Adenovirus Serotype 4 Infection}

\author{
Haiying Ma and Yujuan Niu* \\ The Biomedical Sciences Institute of Qingdao University (Qingdao Branch of SJTU Bio-X Institutes), Qingdao University, \\ Qingdao, China
}

Highly pathogenic fowl adenovirus serotype 4 (FAdV-4) is the causative agent of hydropericardium syndrome (HPS), which is characterized by pericardial effusion and hepatitis, and is one of the foremost causes of economic losses to the poultry industry over the last 30 years. However, the metabolic changes in cells in response to FAdV-4 infection remain unclear. In order to understand the metabolic interactions between the host cell and virus, we utilized ultra-high-performance liquid chromatography/quadrupole time-of-flight tandem mass spectrometry to analyze the metabolic profiles with hepatocellular carcinoma cell line $(\mathrm{LMH})$ infected with FAdV-4. The results showed that FAdV-4 could restore metabolic networks in LMH cells and tricarboxylic acid cycle, glycolysis, and metabolism of purines, pyrimidines, alanine, aspartate, glutamate, and amino sugar and nucleotide sugar moieties. Moreover, FAdV-4 production was significantly reduced in LMH cells cultured in glucose or glutamine-deficient medium. These observations highlighted the importance of host cell metabolism in virus replication. Therefore, similarities and disparities in FAdV-4regulation of the metabolism of host cells could help improve targeted drug and reduce infection.

Keywords: metabolomics, FAdV-4, LMH cells, UHPLC-QTOF-MS, glycolysis, glutamine metabolism

\section{INTRODUCTION}

Fowl adenovirus serotype 4 (FAdV-4) is a highly pathogenic hepatotropic virus and the etiological agent of hydropericardium syndrome (HPS), which is an infectious disease of broiler chickens characterized by pericardial effusion and acute hemorrhagic hepatitis, resulting in high mortality rates (Schachner et al., 2014; Steer et al., 2015; Zhao et al., 2015; Niu et al., 2018a,b; Wang and Zhao, 2019). Although the diagnostic criteria for HPS are relatively well established, the intrinsic mechanisms underlying FAdV-4 infection of host cells remain unclear.

To elucidate the molecular mechanisms underlying the pathogenesis of FAdV-4 infection, high throughput techniques can be used to reveal global changes in the expression profiles of gene and proteins associated with metabolism and disease progression. Metabolomics is a powerful tool to analyze changes to molecular metabolites caused by viral infection in order to clarify 
the interactions between host cells and viruses (Lu et al., 2012). Mounting evidence indicates that similarities and disparities in virus-induced regulation of host cell metabolism could help to improve the efficacy of targeted drug therapies and reduce the incidence of infection (Cui et al., 2013; Sun et al., 2013; Ni et al., 2014). However, relatively few studies have investigated changes to host cell metabolomics in response to FAdV-4 infection. The liver, which is one of the main organs involved in metabolism (Han et al., 2016; Sato et al., 2017; Cappel et al., 2019; Zhou et al., 2020), is the target of FAdV-4 infection. Therefore, metabolomics might be useful to elucidate the pathogenic mechanisms underlying FAdV-4 infection of hepatic cells.

However, there are too many different kinds of cells in the liver to simply clarify the metabolomic changes of hepatocytes, so we chose the hepatocellular carcinoma cell line (LMH) which are often employed as an in vitro model to study the replication and pathogenesis of FAdV-4 (Niu et al., 2019; Zhao et al., 2020). In our study, ultra-high-performance liquid chromatography/quadrupole time-of-flight tandem mass spectrometry (UHPLC-QTOF-MS) was used to assess regulation of the metabolic network of LMH cells infected with FAdV-4 in order to control the onset and progression of HPS.

\section{MATERIALS AND METHODS}

\section{Cell Culture, Virus and Antibodies}

Chicken LMH (ATCC ${ }^{\circledR}$ CRL-2117 ${ }^{\mathrm{TM}}$ ) were obtained from the American Type Culture Collection (Manassas, VA, United States) and cultured as described in a previous study (Niu et al., 2018a). The FAdV-4 strain (SDDM-4/15) used in the study was prepared as described previously (Niu et al., 2016). Self-prepared rabbit polyclonal antibodies against the FAdV-4 hexon protein were used for indirect immunofluorescence (Niu et al., 2018a).

\section{Virus Growth Curve}

LMH cells at $80 \%$ confluence were seeded in the six-well culture plates and infected with FAdV-4 at a multiplicity of infection (MOI) of 1 , while mock-infected cells were treated with an equal volume of Dulbecco's modified Eagle's medium (DMEM). After $2 \mathrm{~h}$, the inoculum was aspirated and the cells were washed twice with phosphate-buffered saline (PBS). Subsequently, the cells were collected at $12,24,36$, and $48 \mathrm{~h}$ post-infection (hpi). A virus growth curve was constructed based on the data acquired by quantitative real-time polymerase chain reaction (qRT-PCR).

\section{Sample Preparation}

At $24 \mathrm{~h}$ before FAdV-4 infection, $\sim 8.0 \times 10^{6} \mathrm{LMH}$ cells were seeded in 100-mm culture plates and cultured to $80-90 \%$ confluence. Twelve plates were prepared, six of which were infected with FAdV-4 at an MOI of 1, while the others were used as the mock group. All cells (about $1.2 \times 10^{7}$ ) were harvested at 24 hpi. Briefly, after the medium was discarded, the cells were washed twice with cold PBS followed by cold $0.9 \%$ sodium chloride solution, then quenched with $1 \mathrm{~mL}$ of methanol:acetonitrile: water $(2: 2: 1, \mathrm{v} / \mathrm{v})$, and stored at $-80^{\circ} \mathrm{C}$ until metabolomics analysis.

\section{Metabolite Extraction and Derivatization}

After thawing, the cell suspensions were sonicated in an ice bath for $30 \mathrm{~min}$ and cooled at $-20^{\circ} \mathrm{C}$ for $10 \mathrm{~min}$ before centrifugation at $14,000 \times g$ for $20 \mathrm{~min}$ at $4^{\circ} \mathrm{C}$. Then, the

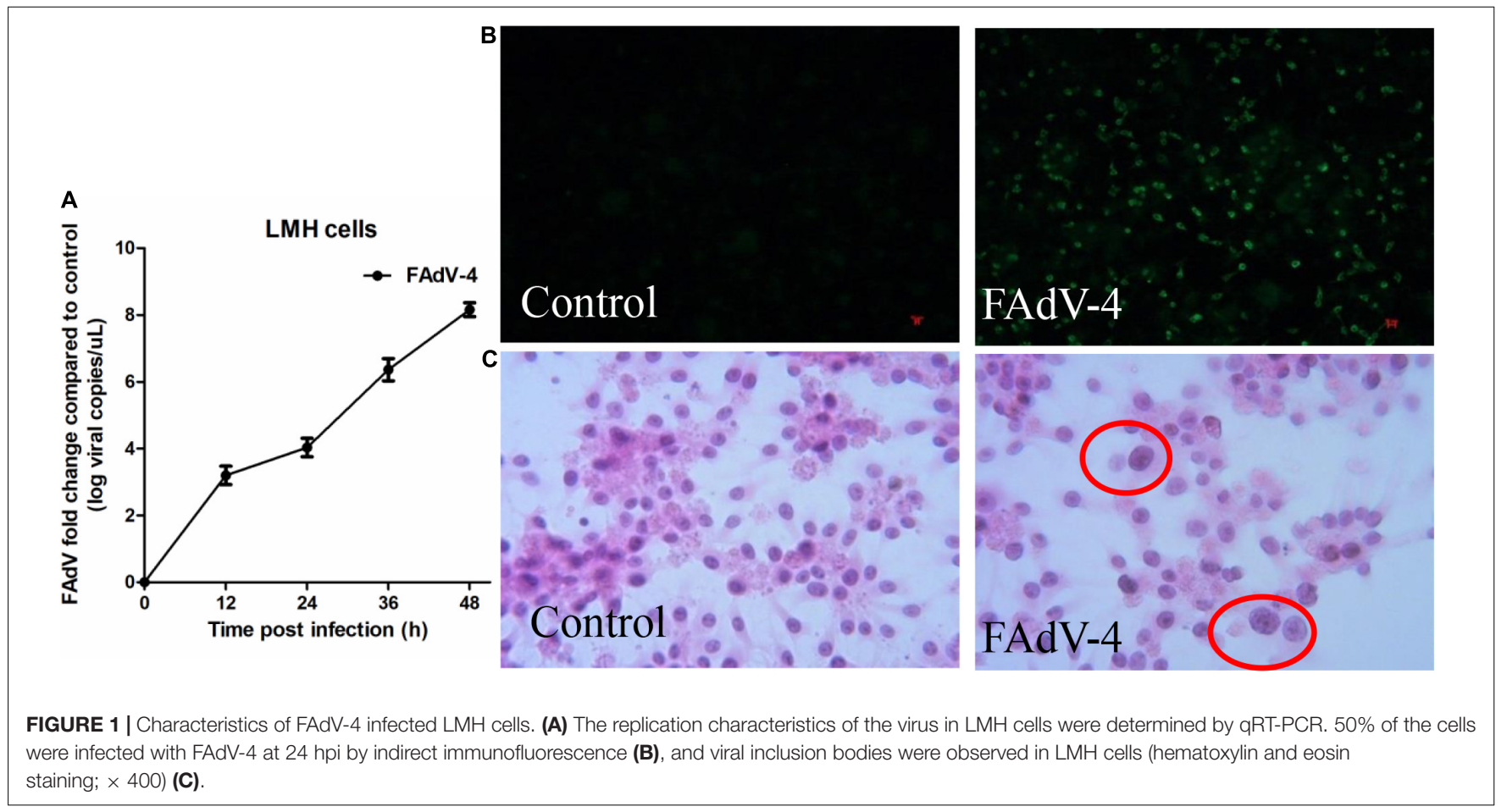



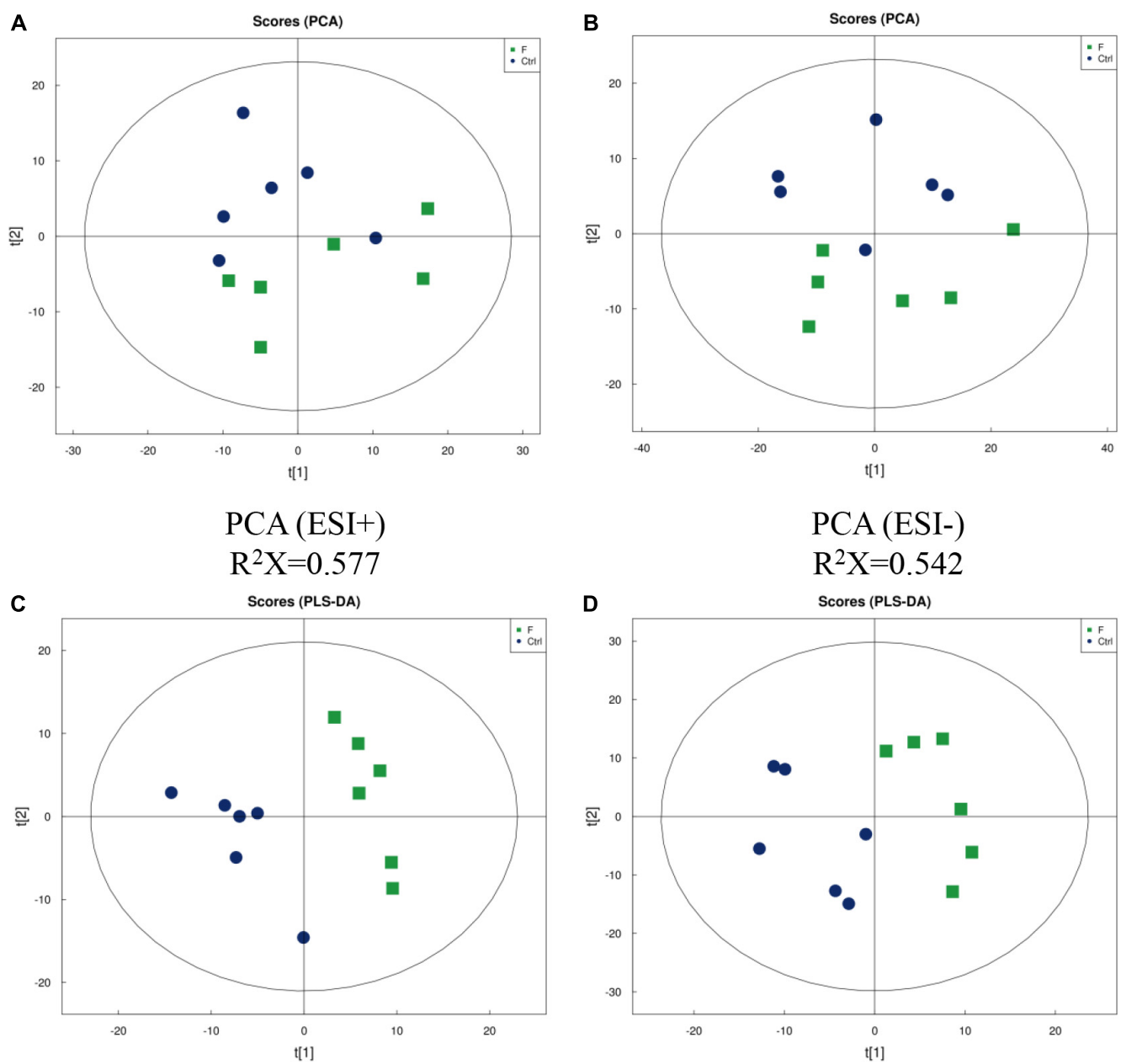

PCA (ESI-)

$\mathrm{R}^{2} \mathrm{X}=0.542$

D
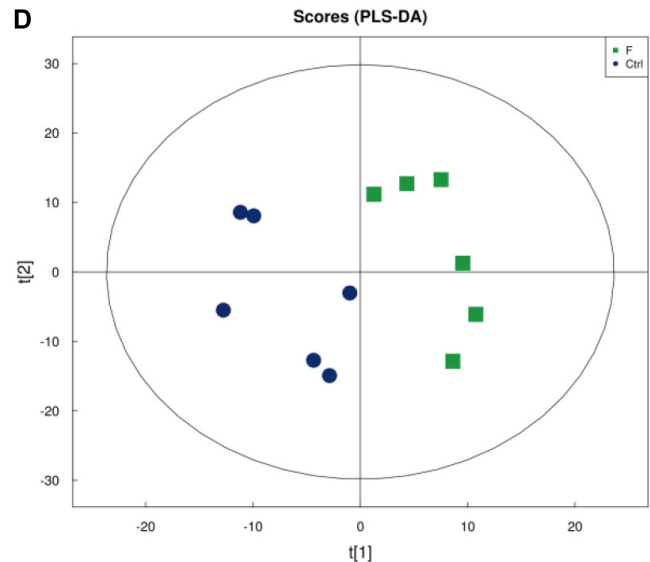

PLS-DA (ESI+)

PLS-DA (ESI-)

$R^{2} \mathrm{X}=0.618, \mathrm{R}^{2} \mathrm{Y}=0.998, \mathrm{Q}^{2}=0.845$

E

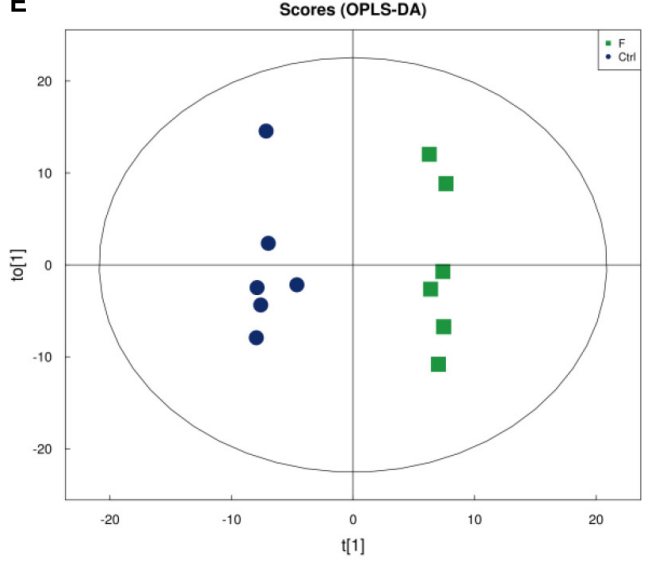

OPLS-DA (ESI +$)$

$R^{2} X=0.522, R^{2} Y=0.984, Q^{2}=0.46$

$\mathbf{F}$

Scores (OPLS-DA)

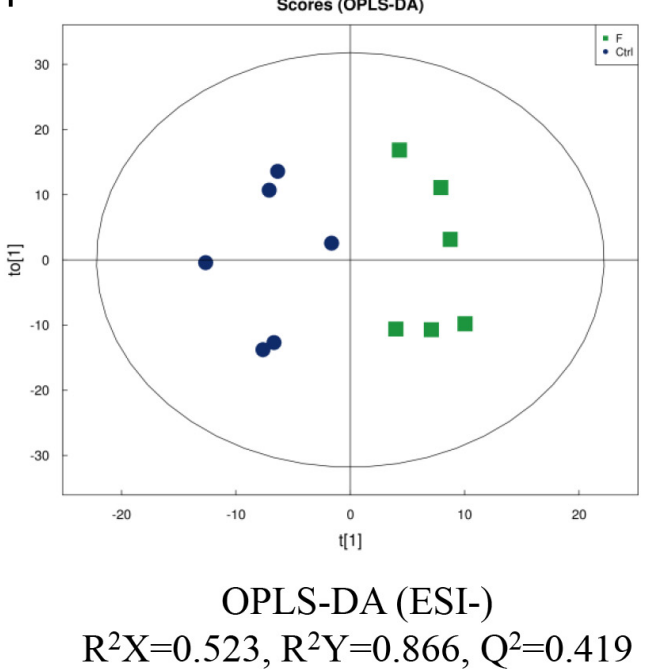

FIGURE 2 | Score plots of PCA, PLS-DA and OPLS-DA of LMH cells infected with FAdV-4. PCA [(A) positive ion mode-ESI + ; (B) negative ion mode-ESI-], PLS-DA [(C) positive ion mode-ESI + ; (D) negative ion mode-ESI-], and OPLS-DA [(E) positive ion mode-ESI + ; (F) negative ion mode-ESI-] models were constructed using LC-MS/MS metabolomics data. Results indicate the separation of control and FAdV-4 group. The ellipses represent $95 \%$ confidence intervals of all samples. 
supernatant was evaporated under a stream of nitrogen gas in a vacuum concentrator. Prior to LC-MS/MS analysis, $100 \mu \mathrm{L}$ of acetonitrile:water solution $(1: 1, \mathrm{v} / \mathrm{v})$ was added to each tube for resolution and the tubes were swirled and centrifuged at $14,000 \times g$ at $4^{\circ} \mathrm{C}$ for $15 \mathrm{~min}$.

\section{LC-MS/MS Analysis}

The samples were assayed using an Agilent 1290 Infinity UHPLC system (Agilent Technologies, Inc., Santa Clara, CA, United States) and a Triple TOF 6600 mass spectrometer (AB SCIEX, Concord, ON, Canada). Electrospray ionization (ESI) in positive and negative ion modes was used to detect the samples with the following parameters: atomization auxiliary heating gas 1, 60 Psi; auxiliary heating gas 2, 60 Psi; curtain gas, 30 Psi; ion source temperature, $600^{\circ} \mathrm{C}$; spray voltage, $\pm 5,500 \mathrm{~V}$ (positive and negative modes); first-grade mass charge ratio detection range, 60-1,000 $\mathrm{Da}$; second grade ion mass charge ratio detection range, $25-1,000 \mathrm{Da}$; first grade mass spectrum scanning accumulation time, $0.20 \mathrm{~s} /$ spectra; and second grade mass spectrum scanning accumulation time, $0.05 \mathrm{~s} /$ spectra. The secondary mass spectrum was obtained in data-dependent acquisition mode with a dynamic exclusion of isotopic ion range of $4 \mathrm{Da}$ with scanning and collecting of 10 debris at a time.

\section{Data Preprocessing and Statistical Analysis}

The raw MS data (wiff.scan files) were converted to MzXML files using ProteoWizard MSConvert software before importing into freely available XCMS software. For peak picking, the following parameters were used: centWave $\mathrm{m} / \mathrm{z}, 25 \mathrm{ppm}$; peakwidth, c (10, $60)$; and prefilter, $c(10,100)$. For peak grouping, the following parameters were used: bw, 5; mzwid, 0.025; and minfrac, 0.5. CAMERA (Collection of Algorithms of MEtabolite pRofile Annotation) was used for annotation of isotopes and adducts. In the extracted ion features, only variables with more than $50 \%$ of non-zero measurement values in at least one group were retained. Compound identification of metabolites was performed by comparing the accuracies of the $\mathrm{m} / \mathrm{z}$ values $(<25 \mathrm{ppm}$ ) and MS/MS spectra with an in-house database established with available authentic standards.

After sum-normalization, the processed data were subjected to multivariate data analysis with the R package "ropls," including

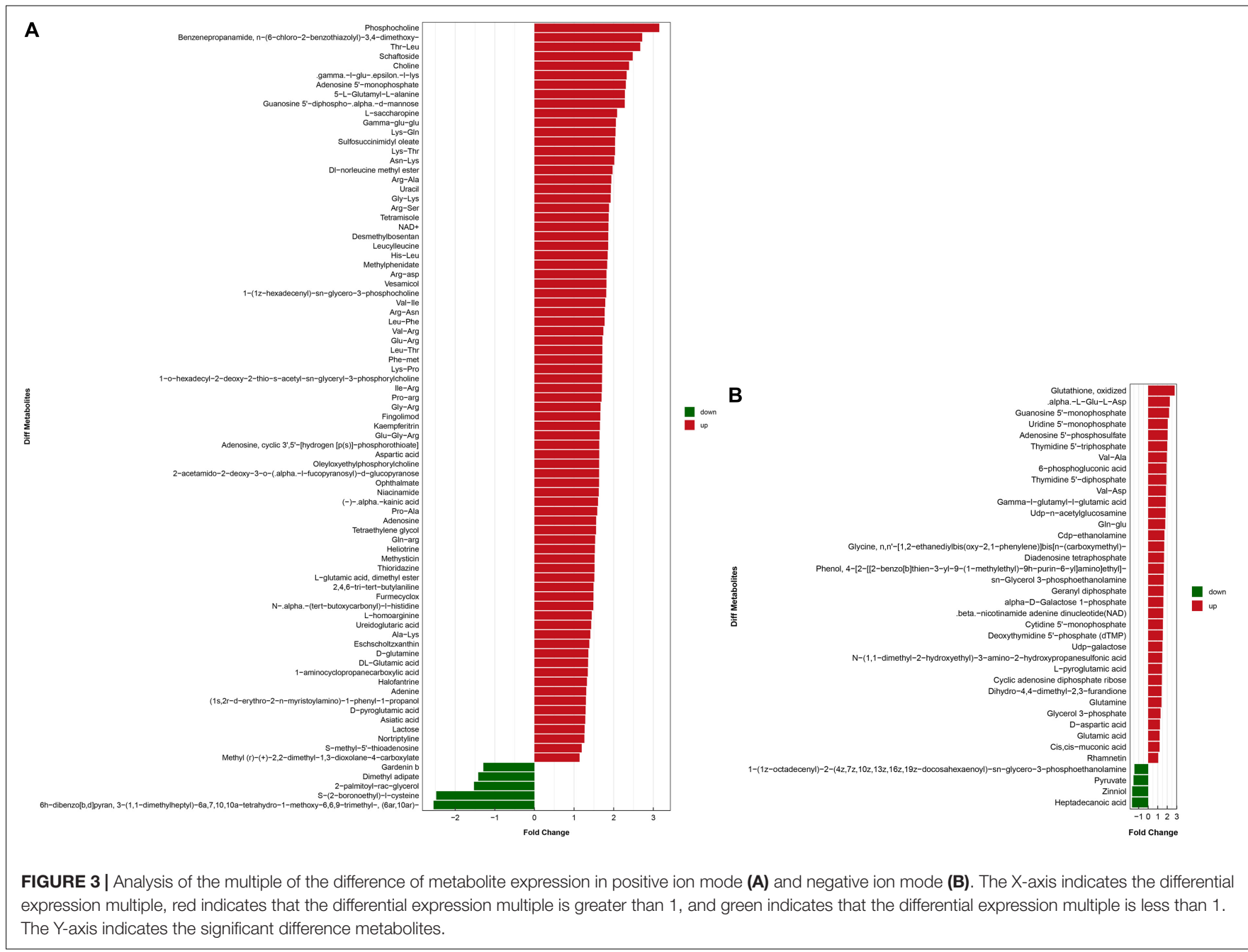


TABLE 1 | Differential metabolites in FAdV-4-infected LMH cells compared to controls in positive ion mode.

\begin{tabular}{|c|c|c|c|c|}
\hline Name & VIP & $P$-value & FC (F vs. Ctrl) & KEGG ID \\
\hline Gly-Arg & 2.19 & 7.79E-07 & 1.67 & \\
\hline Phe-met & 1.02 & 7.80E-07 & 1.71 & \\
\hline Pro-arg & 1.69 & 3.49E-05 & 1.69 & \\
\hline Asn-Lys & 1.44 & 4.11E-05 & 2.02 & \\
\hline Methylphenidate & 1.19 & 6.48E-05 & 1.84 & C07196 \\
\hline Thr-Leu & 1.07 & 6.63E-05 & 2.67 & \\
\hline Leucylleucine & 1.34 & 7.61E-05 & 1.86 & C11332 \\
\hline 2,4,6-tri-tert-butylaniline & 3.71 & 8.32E-05 & 1.49 & \\
\hline His-Leu & 1.01 & 9.90E-05 & 1.85 & C05010 \\
\hline Arg-asp & 1.13 & $1.48 \mathrm{E}-04$ & 1.82 & \\
\hline Pro-Ala & 1.03 & 1.73E-04 & 1.59 & \\
\hline Leu-Thr & 1.17 & $2.20 \mathrm{E}-04$ & 1.71 & \\
\hline Leu-Phe & 1.94 & 2.79E-04 & 1.77 & \\
\hline Arg-Ser & 2.01 & 3.30E-04 & 1.88 & \\
\hline .gamma.-I-glu-.epsilon.-I-lys & 1.45 & $3.78 \mathrm{E}-04$ & 2.33 & $\mathrm{C} 21730$ \\
\hline Lys-Pro & 1.36 & 3.93E-04 & 1.71 & \\
\hline Glu-Arg & 1.68 & 4.69E-04 & 1.72 & \\
\hline Ureidoglutaric acid & 1.95 & 4.90E-04 & 1.43 & \\
\hline Choline & 1.33 & 5.09E-04 & 2.39 & C00114 \\
\hline Adenosine 5'-monophosphate & 10.4 & 5.81E-04 & 2.31 & $\mathrm{C} 00020$ \\
\hline Desmethylbosentan & 1.54 & $6.12 \mathrm{E}-04$ & 1.86 & \\
\hline 1-(1z-hexadecenyl)-sn-glycero-3-phosphocholine & 1.65 & 8.45E-04 & 1.81 & \\
\hline Val-Ile & 1.21 & 9.01E-04 & 1.79 & \\
\hline Val-Arg & 1.46 & 9.37E-04 & 1.74 & \\
\hline 1-o-hexadecyl-2-deoxy-2-thio-s-acetyl-sn-glyceryl-3-phosphorylcholine & 13.5 & 9.84E-04 & 1.71 & \\
\hline Nortriptyline & 1.03 & $1.01 \mathrm{E}-03$ & 1.26 & C07274 \\
\hline $\mathrm{NAD}^{+}$ & 2.20 & 1.02E-03 & 1.87 & C00003 \\
\hline Sulfosuccinimidyl oleate & 7.85 & $1.08 \mathrm{E}-03$ & 2.03 & \\
\hline Fingolimod & 1.34 & $1.42 \mathrm{E}-03$ & 1.66 & \\
\hline Eschscholtzxanthin & 1.13 & 1.49E-03 & 1.38 & C08593 \\
\hline Gly-Lys & 1.08 & $1.56 \mathrm{E}-03$ & 1.92 & \\
\hline Gamma-glu-glu & 7.01 & 1.65E-03 & 2.06 & \\
\hline Niacinamide & 3.88 & 1.67E-03 & 1.62 & C00153 \\
\hline Benzenepropanamide, n-(6-chloro-2-benzothiazolyl)-3,4-dimethoxy- & 2.48 & $1.74 \mathrm{E}-03$ & 2.72 & \\
\hline Phosphocholine & 8.26 & $1.75 \mathrm{E}-03$ & 3.15 & C00588 \\
\hline Heliotrine & 1.09 & $1.86 \mathrm{E}-03$ & 1.53 & C10324 \\
\hline Arg-Ala & 3.86 & $1.99 \mathrm{E}-03$ & 1.94 & \\
\hline Aspartic acid & 1.59 & 2.93E-03 & 1.64 & C00049 \\
\hline Methysticin & 6.17 & $3.10 \mathrm{E}-03$ & 1.52 & C09952 \\
\hline L-saccharopine & 1.18 & $3.40 \mathrm{E}-03$ & 2.09 & C00449 \\
\hline L-glutamic acid, dimethyl ester & 1.50 & $3.44 \mathrm{E}-03$ & 1.51 & \\
\hline Adenosine, cyclic $3^{\prime}, 5^{\prime}$-[hydrogen [p(s)]-phosphorothioate] & 1.31 & 3.68E-03 & 1.64 & \\
\hline Ala-Lys & 1.85 & 4.18E-03 & 1.41 & \\
\hline D-glutamine & 4.64 & $4.22 \mathrm{E}-03$ & 1.36 & C00064 \\
\hline Oleyloxyethylphosphorylcholine & 1.62 & 4.32E-03 & 1.63 & \\
\hline Schaftoside & 5.81 & 4.46E-03 & 2.48 & C10181 \\
\hline 5-L-Glutamyl-L-alanine & 1.63 & 4.60E-03 & 2.28 & C03740 \\
\hline Lactose & 4.92 & 4.71E-03 & 1.27 & $\mathrm{C} 00243$ \\
\hline Glu-Gly-Arg & 2.90 & 4.74E-03 & 1.64 & \\
\hline Lys-Gln & 1.73 & 5.05E-03 & 2.05 & \\
\hline S-(2-boronoethyl)-I-cysteine & 1.64 & 5.59E-03 & 0.40 & \\
\hline Guanosine 5'-diphospho-.alpha.-d-mannose & 1.91 & 5.69E-03 & 2.28 & C00096 \\
\hline L-homoarginine & 1.34 & $6.45 \mathrm{E}-03$ & 1.45 & C01924 \\
\hline
\end{tabular}


TABLE 1 | (Continued)

\begin{tabular}{|c|c|c|c|c|}
\hline Name & VIP & $P$-value & FC (F vs. Ctrl) & KEGG ID \\
\hline Asiatic acid & 1.50 & $6.67 \mathrm{E}-03$ & 1.28 & C08617 \\
\hline Uracil & 1.18 & 7.46E-03 & 1.93 & C00106 \\
\hline 1-aminocyclopropanecarboxylic acid & 1.21 & 7.53E-03 & 1.35 & C01234 \\
\hline DI-norleucine methyl ester & 1.05 & 8.40E-03 & 1.97 & \\
\hline (-)-.alpha.-kainic acid & 1.76 & 9.11E-03 & 1.61 & C12819 \\
\hline (1s,2r-d-erythro-2-n-myristoylamino)-1-phenyl-1-propanol & 1.14 & $9.42 \mathrm{E}-03$ & 1.30 & \\
\hline DL-Glutamic acid & 3.29 & 1.05E-02 & 1.35 & $\mathrm{C} 00025$ \\
\hline Tetramisole & 1.65 & $1.08 \mathrm{E}-02$ & 1.87 & \\
\hline Gardenin b & 1.84 & 1.26E-02 & 0.78 & C15109 \\
\hline Furmecyclox & 1.70 & 1.29E-02 & 1.49 & C18912 \\
\hline 2-palmitoyl-rac-glycerol & 1.35 & $1.32 \mathrm{E}-02$ & 0.66 & \\
\hline 2-acetamido-2-deoxy-3-o-(.alpha.---fucopyranosyl)-d-glucopyranose & 1.39 & 1.35E-02 & 1.63 & \\
\hline D-pyroglutamic acid & 2.70 & 1.38E-02 & 1.29 & $\mathrm{C} 02237$ \\
\hline Tetraethylene glycol & 1.15 & 1.43E-02 & 1.56 & \\
\hline 6h-dibenzo[b,d]pyran, 3-(1,1-dimethylheptyl)-6a,7,10,10a-tetrahydro-1-methoxy-6,6,9- trimethyl-, (6ar,10ar)- & 1.15 & 1.49E-02 & 0.39 & \\
\hline Thioridazine & 1.61 & $1.52 \mathrm{E}-02$ & 1.52 & \\
\hline lle-Arg & 1.83 & $1.53 \mathrm{E}-02$ & 1.70 & \\
\hline Vesamicol & 1.09 & 1.83E-02 & 1.82 & \\
\hline Adenosine & 4.76 & 2.09E-02 & 1.56 & $\mathrm{C} 00212$ \\
\hline Lys-Thr & 1.05 & $2.14 \mathrm{E}-02$ & 2.03 & \\
\hline Adenine & 2.54 & 2.58E-02 & 1.31 & C00147 \\
\hline Halofantrine & 1.87 & 2.71E-02 & 1.33 & C07634 \\
\hline Ophthalmate & 2.10 & 3.06E-02 & 1.63 & C21016 \\
\hline S-methyl-5'-thioadenosine & 3.63 & 3.42E-02 & 1.19 & $\mathrm{C} 00170$ \\
\hline N-.alpha.-(tert-butoxycarbonyl)-I-histidine & 3.48 & 4.22E-02 & 1.49 & \\
\hline Gln-arg & 1.05 & 4.37E-02 & 1.53 & \\
\hline Kaempferitrin & 1.04 & 4.45E-02 & 1.65 & C16981 \\
\hline Methyl (r)-(+)-2,2-dimethyl-1,3-dioxolane-4-carboxylate & 1.33 & 4.46E-02 & 1.14 & \\
\hline Arg-Asn & 1.01 & 4.47E-02 & 1.77 & \\
\hline Dimethyl adipate & 1.74 & 4.74E-02 & 0.71 & C14570 \\
\hline
\end{tabular}

Name is the name of the metabolite.

FC (fold changes) is the difference multiple.

KEGG ID is the KEGG number of the metabolite.

E-a represents 10^a in P-value.

pareto-scaled principal component analysis (PCA), partial least squares discrimination analysis (PLS-DA), and orthogonal partial least-squares discriminant analysis (OPLS-DA). Sevenfold crossvalidation and response permutation testing was used to evaluate the robustness of the model. The variable importance in the projection (VIP) value of each variable in the PLS-DA and OPLSDA models was calculated to indicate potential contribution to the classification. The quality of the models is described by the $\mathrm{R}^{2} \mathrm{X}$ or $\mathrm{R}^{2} \mathrm{Y}$ and $\mathrm{Q}^{2}$ values. The Student's $t$-test was applied to determine the significance of differences between two groups of independent samples. VIP $>1$ and a probability $(p)$ value $<0.05$ were used to screen for significant changes in metabolites. Pearson's correlation analysis was performed to identify potential correlations between two variables.

\section{Inhibitory Effects of Related Metabolic Pathways on FAdV-4 Replication}

To investigate the impact of host cell metabolism of glucose and glutamine on FAdV-4 replication, $\mathrm{LMH}$ cells were cultured in DMEM without glucose or glutamine. As drug treatment samples, FAdV-4-infected LMH cells were treated with 10 or $40 \mu \mathrm{M}$ 2-deoxy-D-glucose (2dGlc) (a commonly used inhibitor of glycolysis), 1 or $10 \mu \mathrm{M}$ CB-839, or dimethyl sulfoxide (DMSO) for $48 \mathrm{~h}$. Cells in the DMSO group were infected with FAdV-4 and cultured in DMEM supplemented with glucose and glutamine. Cells were lysed for western blot analysis.

\section{RESULTS}

\section{Characteristics of FAdV-4 Infected LMH Cells}

FAdV-4 is known to cause cytopathologic effects in LMH cells. Firstly, the replication characteristics of the virus in LMH cells were determined by qRT-PCR. As shown in Figure 1A, virus titers in LMH cells reached $10^{4}$ at $24 \mathrm{hpi}$. Indirect immunofluorescence showed that about $50 \%$ of the cells were infected with FAdV-4 at 24 hpi (Figure 1B), and hematoxylin and eosin staining confirmed the formation of 
viral inclusion bodies (Figure 1C). Combined with previous research results (Niu et al., 2018a), FAdV-4 could cause significant apoptosis, autophagy, and a severe inflammatory response in LMH cells at 24 hpi. Therefore, mock and FAdV4-infected LMH cells were prepared at $24 \mathrm{hpi}$ for early metabolomics analysis.

\section{Analysis of Metabolomics Based on LC-MS/MS}

The metabolomic profiles of LMH cells infected with FAdV4 were characterized using LC-MS/MS techniques, and PCA,
PLS-DA, and OPS-DA were applied to the data. Analysis of data quality, including total ion chromatogram, PCA, Pearson's correlation, Hotelling's $\mathrm{t}^{2}$ statistic, multivariate control chart, and relative standard deviation, indicated that the quality of data was acceptable for the following metabolomics analysis. PCA score plots revealed clear separation between the control and FAdV-4-infected groups, under both positive and negative modes (Figures 2A,B). The LC-MS/MS data were further subjected to PLS-DA and OPLS-DA. The quality of the PLS-DA and OPLS-DA models was evaluated based on the parameters $R^{2} Y$ and $\mathrm{Q}^{2}$, which are measures of fitness and prediction ability,

TABLE 2 | Differential metabolites in FAdV-4-infected LMH cells compared to controls in negative ion mode.

\begin{tabular}{|c|c|c|c|c|}
\hline Name & VIP & $P$-value & FC (F vs. Ctrl) & KEGG ID \\
\hline Val-Ala & 1.03 & $1.01 \mathrm{E}-04$ & 1.96 & \\
\hline Adenosine 5'-phosphosulfate & 5.84 & $5.41 E-04$ & 2.04 & C00224 \\
\hline Guanosine 5'-monophosphate & 2.03 & $1.20 \mathrm{E}-03$ & 2.20 & $\mathrm{C} 00144$ \\
\hline Deoxythymidine 5'-phosphate (dTMP) & 1.14 & $1.47 \mathrm{E}-03$ & 1.54 & $\mathrm{C} 00364$ \\
\hline beta.-nicotinamide adenine dinucleotide (NAD) & 1.04 & $1.52 \mathrm{E}-03$ & 1.58 & C00003 \\
\hline Gamma-I-glutamyl-I-glutamic acid & 3.53 & 2.69E-03 & 1.84 & C05282 \\
\hline .alpha.-L-Glu-L-Asp & 2.92 & $2.78 \mathrm{E}-03$ & 2.27 & \\
\hline Uridine $5^{\prime}$-monophosphate & 7.41 & 3.03E-03 & 2.05 & C00105 \\
\hline Cdp-ethanolamine & 1.63 & 4.19E-03 & 1.71 & C00570 \\
\hline Cyclic adenosine diphosphate ribose & 2.07 & 4.53E-03 & 1.44 & C13050 \\
\hline Thymidine $5^{\prime}$-triphosphate & 1.78 & 5.38E-03 & 2.00 & C00459 \\
\hline Val-Asp & 1.29 & $6.41 \mathrm{E}-03$ & 1.87 & \\
\hline Heptadecanoic acid & 1.47 & $6.91 \mathrm{E}-03$ & 0.59 & \\
\hline Glutamine & 4.79 & 1.00E-02 & 1.40 & C00064 \\
\hline Diadenosine tetraphosphate & 1.02 & $1.02 \mathrm{E}-02$ & 1.66 & \\
\hline Geranyl diphosphate & 1.67 & 1.05E-02 & 1.61 & $\mathrm{C} 05847$ \\
\hline 6-phosphogluconic acid & 1.03 & 1.07E-02 & 1.91 & $\mathrm{C} 00345$ \\
\hline Udp-n-acetylglucosamine & 12.1 & $1.14 \mathrm{E}-02$ & 1.82 & $\mathrm{C} 00043$ \\
\hline Dihydro-4,4-dimethyl-2,3-furandione & 2.30 & $1.54 \mathrm{E}-02$ & 1.41 & $\mathrm{C} 01125$ \\
\hline Gln-glu & 2.31 & $2.18 \mathrm{E}-02$ & 1.80 & \\
\hline Thymidine $5^{\prime}$-diphosphate & 2.13 & 2.23E-02 & 1.91 & $\mathrm{C} 00363$ \\
\hline Pyruvate & 1.37 & 2.30E-02 & 0.65 & $\mathrm{C} 00022$ \\
\hline alpha-D-Galactose 1-phosphate & 3.09 & 2.46E-02 & 1.59 & $\mathrm{C} 00103$ \\
\hline N-(1,1-dimethyl-2-hydroxyethyl)-3-amino-2-hydroxypropanesulfonic acid & 1.18 & 2.66E-02 & 1.48 & \\
\hline 1-(1z-octadecenyl)-2-(4z,7z,10z,13z,16z,19z-docosahexaenoyl)-sn-glycero-3-phosphoethanolamine & 2.21 & 2.85E-02 & 0.69 & \\
\hline Cis, cis-muconic acid & 9.05 & $2.90 \mathrm{E}-02$ & 1.20 & $\mathrm{C} 02480$ \\
\hline Zinniol & 2.73 & $2.99 \mathrm{E}-02$ & 0.60 & C10840 \\
\hline Glycine, n, n'-[1,2-ethanediylbis(oxy-2,1-phenylene)]bis[n-(carboxymethyl)- & 2.60 & $3.23 E-02$ & 1.68 & \\
\hline sn-Glycerol 3-phosphoethanolamine & 3.80 & $3.44 \mathrm{E}-02$ & 1.62 & \\
\hline D-aspartic acid & 3.19 & $3.51 \mathrm{E}-02$ & 1.23 & $\mathrm{C} 00402$ \\
\hline Phenol,4-[2-[[2-benzo[b]thien-3-yl-9-(1-methylethyl)-9h-purin-6-yl]amino]ethyl]- & 1.25 & $3.58 \mathrm{E}-02$ & 1.65 & \\
\hline Glycerol 3-phosphate & 1.35 & $3.74 \mathrm{E}-02$ & 1.30 & C00093 \\
\hline Udp-galactose & 2.14 & 3.81E-02 & 1.50 & C00052 \\
\hline Cytidine $5^{\prime}$-monophosphate & 1.25 & $3.92 \mathrm{E}-02$ & 1.55 & C00055 \\
\hline Rhamnetin & 2.63 & 4.18E-02 & 1.05 & C10176 \\
\hline L-pyroglutamic acid & 3.53 & 4.48E-02 & 1.45 & C01879 \\
\hline Glutathione, oxidized & 9.09 & 4.76E-02 & 2.78 & $\mathrm{C} 00127$ \\
\hline Glutamic acid & 4.00 & 4.99E-02 & 1.20 & C00025 \\
\hline
\end{tabular}

Name is the name of the metabolite.

$F C$ (fold changes) is the difference multiple.

KEGG ID is the KEGG number of the metabolite.

$E$-a represents 10^a in $P$-value. 
respectively. Generally, $\mathrm{Q}^{2}$ is greater than 0.5 , indicating that the model is stable and reliable, where $0.3<\mathrm{Q} 2 \leq 0.5$ indicates that the model is stable and Q2 $<0.3$ indicates low reliability of the model. PLS-DA and OPLA-DA score plots revealed excellent separation of the control and FAdV-4-infected groups (Figures 2C-F).

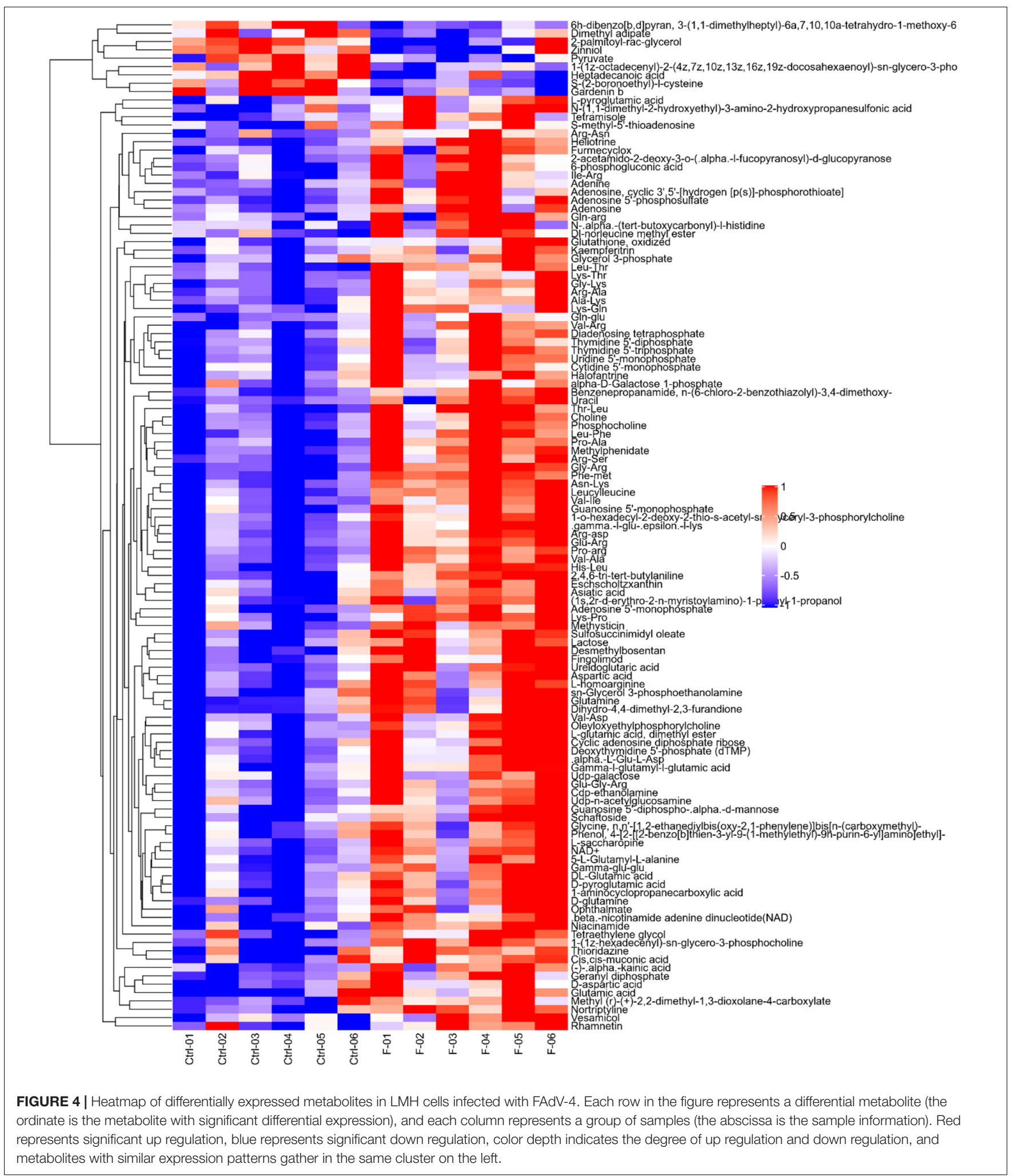




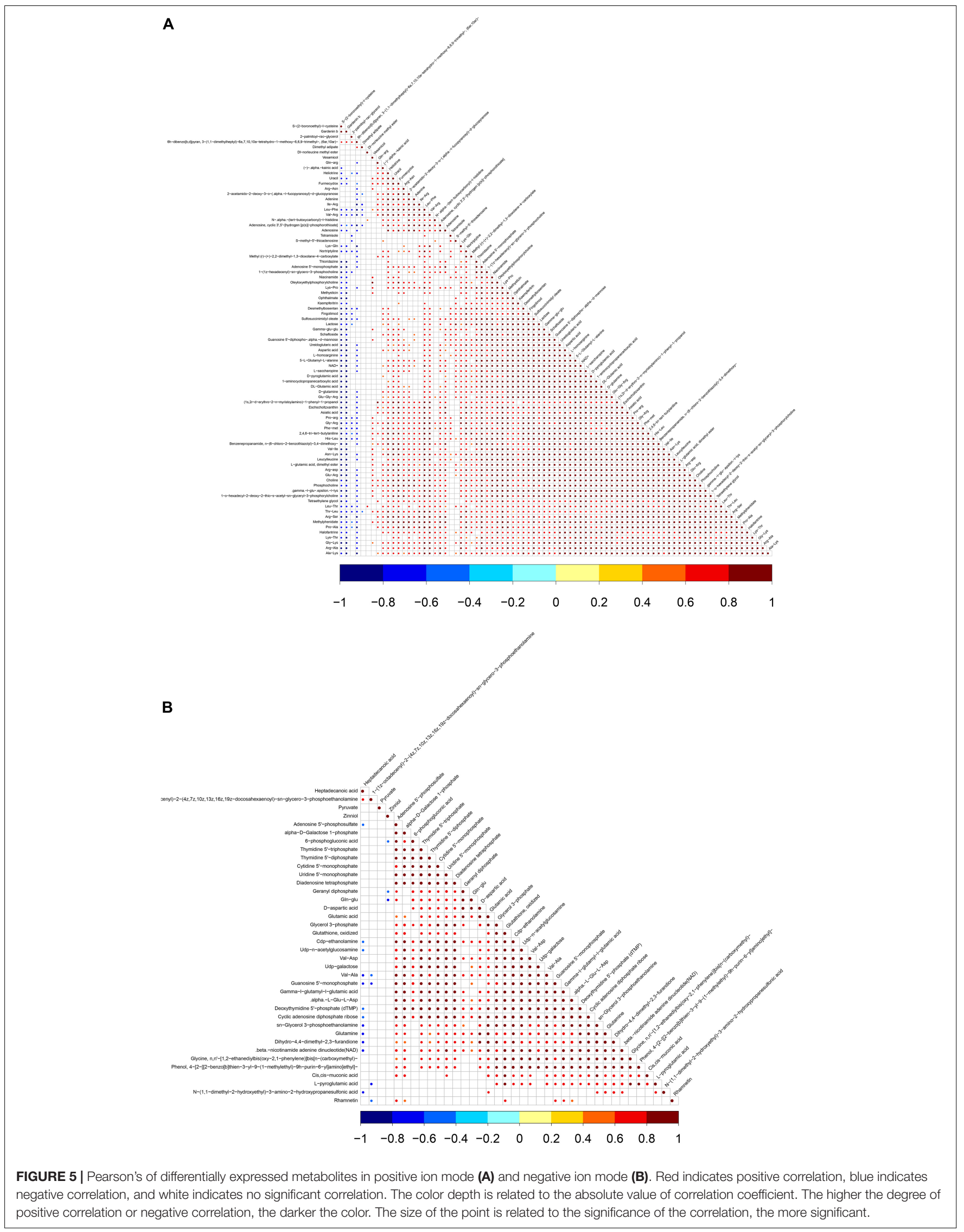




\section{Differential Metabolites}

OPLS-DA based on a VIP $>1$ in and $P<0.05$ (Student's $t$-test) revealed significant differences in metabolite screening criteria. The metabolites and associated fold changes in response to FAdV4 infection of LMH cells are shown in Figure 3 and Tables 1, 2. The differential metabolites, which included 49 amino acids and related derivatives, of LMH cells caused by FAdV-4 infection, included 78 that were increased and five that were decreased in positive ion mode, while 34 were increased and four were decreased in negative ion mode.

\section{Changes to Metabolic Pathways}

Differential metabolites were further analyzed to explore the effects of FAdV-4 infection on the metabolic pathways of LMH cells. The relationships among the differential metabolites were investigated using heatmap analysis (Figure 4) and Pearson's correlation (Figure 5). The results indicated that metabolites in the same or related metabolic pathways were closely connected. When the significance level of metabolite enrichment in each pathway was analyzed and calculated with the Fisher's exact test, 60 pathways were potentially changed in LMH cells infected with FAdV-4 (Table 3). The top 20 metabolic pathways with the highest significance were selected based on $P$ values and presented in the form of a histogram (Figure 6). The top 20 metabolic pathways mainly involved pyrimidine metabolism, purine metabolism, amino acid metabolism, carbohydrate metabolism, glutathione metabolism, and cofactor and vitamin metabolism, in addition to some signal transduction pathways. Next, some metabolites with significant differences were further screened to establish connections among the related metabolic pathways (Figure 7). The results showed that the tricarboxylic acid (TCA) cycle, purine metabolism, pyrimidine metabolism, alanine, aspartate, and glutamate metabolism, amino sugar and nucleotide sugar metabolism, and glycolysis were significantly up-regulated and closely connected.

\section{Glucose and Glutamine Metabolism on FAdV-4 Replication}

As shown in Figure 8, treatment of LMH cells with either glutamine withdrawal or 1 or $10 \mu \mathrm{M}$ CB-839 significantly reduced FAdV-4 replication and lowered viral yields relative to DMSO treatment in the presence of $4 \mathrm{mM}$ glutamine. Moreover, the inhibitory effect of CB-839 on FAdV-4 was dose-dependent. FAdV-4 production was significantly reduced in LMH cells cultured in glucose-deficient medium. Treatment of FAdV-4infected cells with 20 or $40 \mu \mathrm{M} 2 \mathrm{dGlc}$ also effectively impaired the production of infectious particles.

\section{DISCUSSION}

Viruses, as obligate intracellular parasites, are completely dependent on host cell metabolism for replication, production, and release (Harries et al., 2010; Thaker et al., 2019b). In order to fulfill these requirements, viruses have evolved different mechanisms to reprogram and exploit the host
TABLE 3 | Differential metabolites in FAdV-4-infected LMH cells compared to controls in negative ion mode.

\begin{tabular}{|c|c|}
\hline Metabolic pathway & $P$-value \\
\hline Pyrimidine metabolism & 0.00 \\
\hline Neuroactive ligand-receptor interaction & 0.00 \\
\hline Zeatin biosynthesis & 0.00 \\
\hline Purine metabolism & 0.00 \\
\hline Alanine, aspartate and glutamate metabolism & 0.00 \\
\hline FoxO signaling pathway & 0.00 \\
\hline ABC transporters & 0.00 \\
\hline Taste transduction & 0.00 \\
\hline Renin secretion & 0.00 \\
\hline AMPK signaling pathway & 0.00 \\
\hline cGMP-PKG signaling pathway & 0.00 \\
\hline D-Glutamine and D-glutamate metabolism & 0.00 \\
\hline Cysteine and methionine metabolism & 0.00 \\
\hline Oxidative phosphorylation & 0.00 \\
\hline Lysosome & 0.00 \\
\hline Glutathione metabolism & 0.00 \\
\hline Taurine and hypotaurine metabolism & 0.00 \\
\hline Protein digestion and absorption & 0.00 \\
\hline Arginine biosynthesis & 0.00 \\
\hline Glycerophospholipid metabolism & 0.00 \\
\hline Aminoacyl-tRNA biosynthesis & 0.00 \\
\hline Metabolic pathways & 0.00 \\
\hline Biosynthesis of amino acids & 0.00 \\
\hline Nicotinate and nicotinamide metabolism & 0.00 \\
\hline Olfactory transduction & 0.00 \\
\hline Longevity regulating pathway & 0.00 \\
\hline Glutamatergic synapse & 0.00 \\
\hline GABAergic synapse & 0.00 \\
\hline Pantothenate and CoA biosynthesis & 0.00 \\
\hline Longevity regulating pathway -worm & 0.01 \\
\hline beta-Alanine metabolism & 0.01 \\
\hline Calcium signaling pathway & 0.01 \\
\hline Photosynthesis & 0.01 \\
\hline Insulin secretion & 0.01 \\
\hline Synaptic vesicle cycle & 0.01 \\
\hline Monobactam biosynthesis & 0.01 \\
\hline Regulation of lipolysis in adipocytes & 0.01 \\
\hline Platelet activation & 0.01 \\
\hline Bacterial secretion system & 0.01 \\
\hline HIF-1 signaling pathway & 0.01 \\
\hline Galactose metabolism & 0.02 \\
\hline Proximal tubule bicarbonate reclamation & 0.02 \\
\hline Histidine metabolism & 0.02 \\
\hline Glycine, serine and threonine metabolism & 0.02 \\
\hline Nitrogen metabolism & 0.02 \\
\hline Biosynthesis of various secondary metabolites - part 3 & 0.02 \\
\hline Two-component system & 0.02 \\
\hline Aldosterone synthesis and secretion & 0.03 \\
\hline Carbon fixation in photosynthetic organisms & 0.03 \\
\hline Thermogenesis & 0.03 \\
\hline cAMP signaling pathway & 0.03 \\
\hline Glyoxylate and dicarboxylate metabolism & 0.03 \\
\hline Amino sugar and nucleotide sugar metabolism & 0.03 \\
\hline Glucagon signaling pathway & 0.03 \\
\hline Carbon metabolism & 0.04 \\
\hline Ferroptosis & 0.04 \\
\hline mTOR signaling pathway & 0.04 \\
\hline PI3K-Akt signaling pathway & 0.04 \\
\hline Thiamine metabolism & 0.05 \\
\hline Glycolysis/Gluconeogenesis & 0.05 \\
\hline
\end{tabular}

0.00 means that $P$-value is less than 0.01 . 


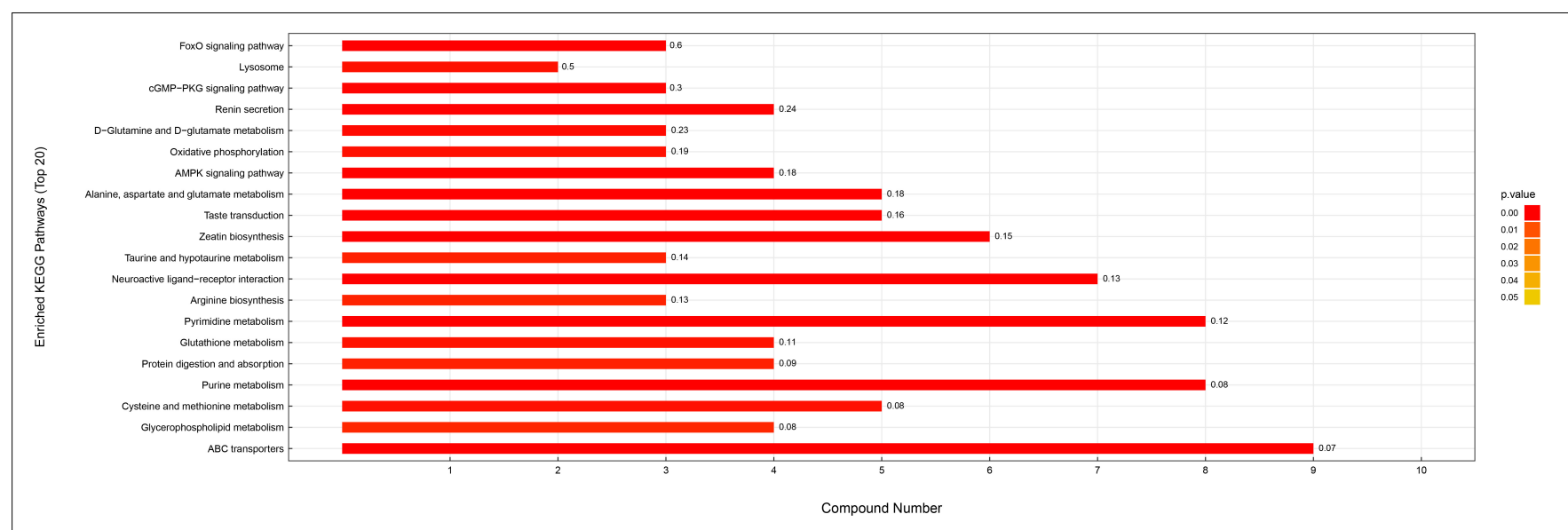

FIGURE 6 | KEGG enrichment pathway map. The vertical axis represents each KEGG metabolic pathway, and the horizontal axis represents the number of differentially expressed metabolites in each KEGG metabolic pathway. Color represents the $P$ value of enrichment analysis. The darker the color is, the smaller the $P$ value is, and the more significant the enrichment degree is. The number on the column represents the proportion of different metabolites in the detected metabolites.

metabolism (Mesquita and Estaquier, 2018). Therefore, understanding changes to the metabolism of virus-infected host cells would be useful to develop targeted therapies. In recent years, metabolomics analysis has been conducted of various viruses, such as Zika virus (Thaker et al., 2019a), Newcastle disease virus (Liu et al., 2019), and classical swine fever virus (Gou et al., 2017). This study is the first to employ metabolomics to analyze changes in metabolites and metabolic pathways in FAdV-4-infected LMH cells, which revealed insights into the mechanisms of the pathogenesis and host cell interactions of FAdV-4.

The results of this study provide useful information on alterations to metabolites and associated pathways in $\mathrm{LMH}$ cells infected with FAdV-4 (Figures 3, 4, 6). Several pathways involved in energy metabolism were found to be perturbed during FAdV-4 infection, including the TCA, purine metabolism, pyrimidine metabolism, glycolysis, and the metabolism of some amino acids. In a sense, changes to these metabolic pathways could reflect the contributions of host cells to virus proliferation.

Glucose oxidation is a major source of carbon and energy in cellular bioprocesses. During glycolysis, glucose is metabolized to pyruvate (Tohyama et al., 2016). In the presence of oxygen or under oxygen-limiting conditions, pyruvate is catabolized in the TCA to generate large amounts of adenosine triphosphate (ATP) or lactate. Increased amounts of glycolytic intermediates provide the precursors required for synthesis of nucleotides, amino acids, and lipids, as well as cellular redox homeostasis (Vander Heiden et al., 2009; DeBerardinis and Chandel, 2016). Metabolomics analysis revealed that FAdV-4 significantly affected glycolysis and, subsequently, the intermediates of purine metabolism. However, FAdV-4 infection did not induce significant changes to the intermediates of the TCA cycle for ATP production, with the exception of citrate. In a previous study, FAdV-4 infection induced swelling of the mitochondria of hepatocytes and the disappearance of cristae. Oxidative phosphorylation and ATP synthesis occur in the mitochondria (Zorova et al., 2018).
However, FAdV-4 infection impairs oxidative phosphorylation, at least to a certain extent, which can result in mitochondrial injury. On the other hand, pyruvate carboxylation will replenish the metabolites involved in the TCA cycle, while redirecting other TCA intermediates to pyrimidine biosynthesis (Vastag et al., 2011). In this study, pyrimidine biosynthesis was increased (Figures 6, 7). The metabolism of purines and pyrimidines can be summarized as nucleic acid metabolism. Increase metabolism of purines and pyrimidines provides materials for virus replication. In general, glycolysis is a key metabolic pathway. Previous studies have shown that glucose metabolism can affect virus replication (Yu et al., 2011; Thai et al., 2014; Kong et al., 2015). The results showed that in glucose-deficient medium, FAdV-4 production was reduced in LMH cells. Later studies showed that treatment of FAdV-4-infected cells with $2 \mathrm{dGlc}$, a commonly used inhibitor of glycolysis, also effectively impaired the production of infectious particles (Figure 8), likely due to reduced glycosylation of viral glycoproteins. Although it is clear that glucose metabolism is important for FAdV-4 infection, the exact mechanisms responsible for virus-induced activation of glycolysis are still not fully understood. Since glucose uptake is important for bioenergy requirements and cell biomass, increased glycolysis may be used by viruses as a source of biomass replication. Increasing glucose uptake may also be required for other metabolic pathways, such as the pentose phosphate and nucleic acid pathways. In fact, virus-induced changes to glucose metabolism seem to play a key role in successful infection, thus it is important to understand the precise molecular mechanisms that drive this reprogramming.

Apart from glucose, glutamine, which is classified as a nonessential amino acid, plays a crucial role in cell proliferation. Virus infection of cells will enhance bioenergetics and macromolecular synthesis, which may include glutamine uptake and utilization. Glutamine serves as a carbon source to support the biosynthesis of lipids, amino acids, and nucleotides (Lunt and Vander Heiden, 2011; DeBerardinis and Chandel, 2016). 


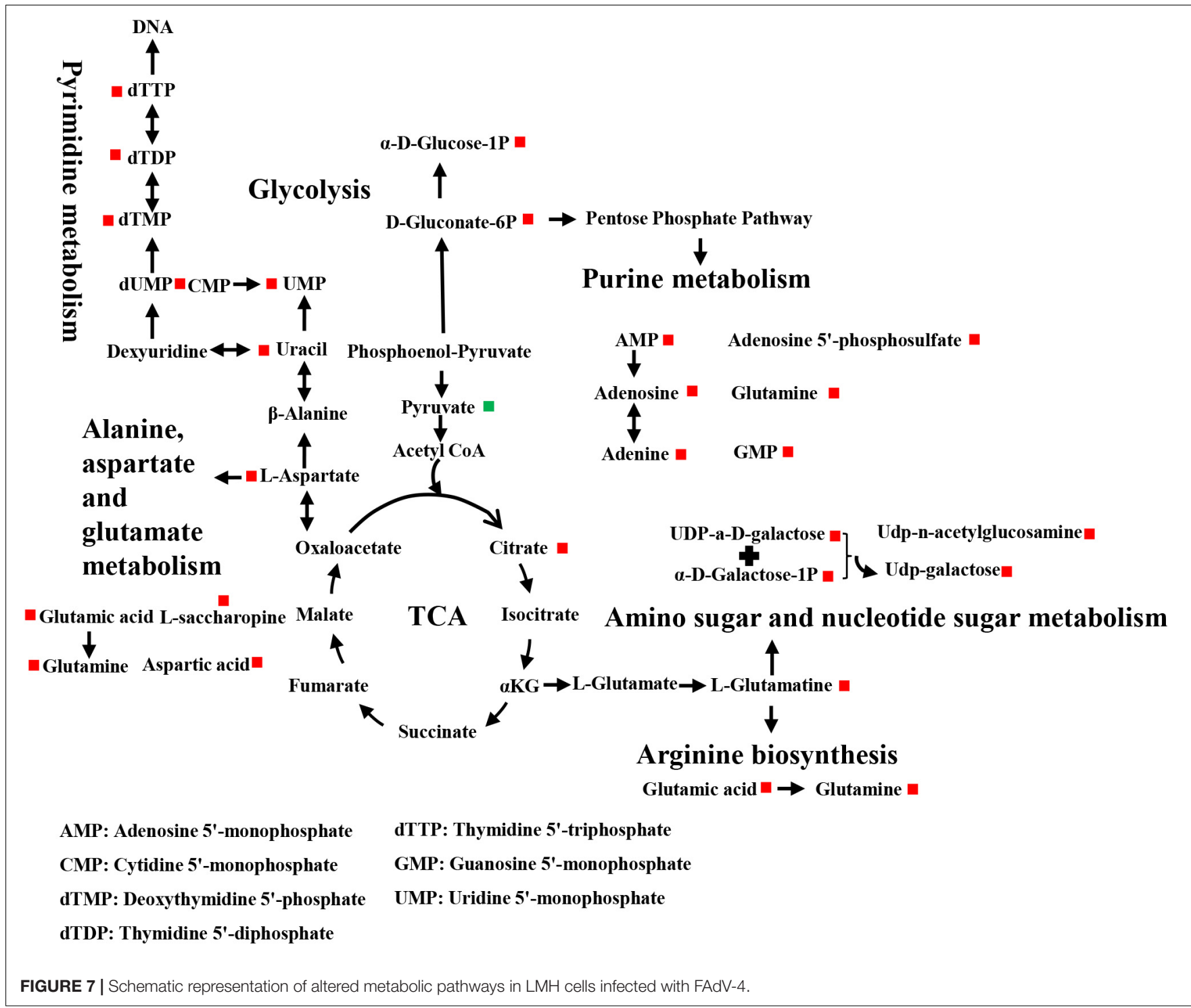

Therefore, glutamine availability is essential for the replication of several viruses. In this study, glutamine metabolism was notably increased in response to FAdV-4 infection

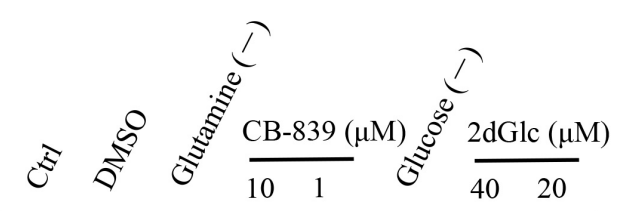

Hexon

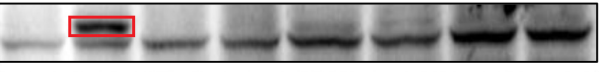

GAPDH

FIGURE 8 | Western blot analysis hexon expression in LMH cells by affecting glucose and glutamine metabolism. The destination strip is marked in the red box, and the below bands are non-specific.
(Figures 6, 7). Moreover, FAdV-4 replication was found to be glutamine-dependent as cultivation in glutamine-free medium completely abolished the production of infectious viral particles. Treatment of LMH cells with 1.0 or $10 \mu \mathrm{M}$ CB-839 significantly decreased replication of FAdV-4 as compared with DMSO treatment (Figure 8). This observation highlights the importance of glutamine in virus replication, although future studies are needed to further clarify the underlying molecular mechanisms.

FAdV-4 infection caused changes to a variety of metabolic pathways, among which the metabolism of purines and pyrimidines is particularly important. It is a known that virus infection affects nucleotide metabolism of the host cells in order to meet the nucleic acid needs of the virus (Munger et al., 2006; Vastag et al., 2011; Thai et al., 2014). In this study, the concentrations of the intermediates of the purine and pyrimidine biosynthesis pathways had increased (Figure 7). 
In summary, these metabolomics data provide evidence that FAdV-4 restored the metabolic networks in $\mathrm{LMH}$ cells. Various metabolic pathways, including glycolysis and the metabolism of glutamine, amino acids, purines, and pyrimidines, were altered. Hence, virus infection modified the metabolism of the host cell to provide energy and materials for replication. The identification of these changes will provide considerable important information for further understanding of FAdV-4 replication, pathogenesis, and drug sensitivity.

\section{DATA AVAILABILITY STATEMENT}

The original contributions presented in the study are included in the article/supplementary material, further inquiries can be directed to the corresponding author/s.

\section{REFERENCES}

Cappel, D. A., Deja, S., Duarte, J. A. G., Kucejova, B., Inigo, M., Fletcher, J. A., et al. (2019). Pyruvate-carboxylase-mediated anaplerosis promotes antioxidant capacity by sustaining TCA cycle and redox metabolism in liver. Cell Metab. 29, 1291.e8-1305.e8. doi: 10.1016/j.cmet.2019.03.014

Cui, L., Lee, Y. H., Kumar, Y., Xu, F. G., Lu, K., Ooi, E. E., et al. (2013). Serum metabolome and lipidome changes in adult patients with primary dengue infection. PLoS Negl. Trop. Dis. 7:e2373. doi: 10.1371/journal.pntd.000 2373

DeBerardinis, R. J., and Chandel, N. S. (2016). Fundamentals of cancer metabolism. Sci. Adv. 2:e1600200. doi: 10.1126/sciadv.1600200

Gou, H., Zhao, M., Yuan, J., Xu, H., Ding, H., and Chen, J. (2017). Metabolic profiles in cell lines infected with classical swine fever virus. Front. Microbiol. 8:691.

Han, H. S., Kang, G., Kim, J. S., Choi, B. H., and Koo, S. H. (2016). Regulation of glucose metabolism from a liver-centric perspective. Exp. Mol. Med. 48:e218. doi: 10.1038/emm.2015.122

Harries, P. A., Schoelz, J. E., and Nelson, R. S. (2010). Intracellular transport of viruses and their components: utilizing the cytoskeleton and membrane highways. Mol. Plant Microbe Interact. 23, 1381-1393. doi: 10.1094/MPMI-0510-0121

Kong, K., Kumar, M., Taruishi, M., and Javier, R. T. (2015). Adenovirus E4-ORF1 dysregulates epidermal growth factor and insulin/insulin-like growth factor receptors to mediate constitutive myc expression. J. Virol. 89, 10774-10785. doi: 10.1128/JVI.01463-15

Liu, P., Yin, Y., Gong, Y., Qiu, X., Sun, Y., Tan, L., et al. (2019). In vitro and in vivo metabolomic profiling after infection with virulent newcastle disease virus. Viruses 11:962. doi: 10.3390/v11100962

Lu, K., Knutson, C. G., Wishnok, J. S., Fox, J. G., and Tannenbaum, S. R. (2012). Serum metabolomics in a Helicobacter hepaticus mouse model of inflammatory bowel disease reveal important changes in the microbiome, serum peptides, and intermediary metabolism. J. Proteome Res. 11, 4916-4926. doi: 10.1021/ pr300429x

Lunt, S. Y., and Vander Heiden, M. G. (2011). Aerobic glycolysis: meeting the metabolic requirements of cell proliferation. Annu. Rev. Cell Dev. Biol. 27, 441-464. doi: 10.1146/annurev-cellbio-092910-154237

Mesquita, I., and Estaquier, J. (2018). Viral manipulation of the host metabolic network. Exp. Suppl. 109, 377-401. doi: 10.1007/978-3-319-74932-7_10

Munger, J., Bajad, S. U., Coller, H. A., Shenk, T., and Rabinowitz, J. D. (2006). Dynamics of the cellular metabolome during human cytomegalovirus infection. PLoS Pathog. 2:e132. doi: 10.1371/journal.ppat.0020132

Ni, Y., Xie, G. X., and Jia, W. (2014). Metabonomics of human colorectal cancer: new approaches for early diagnosis and biomarker discovery. J. Proteome Res. 13, 3857-3870. doi: 10.1021/pr500443c

\section{AUTHOR CONTRIBUTIONS}

YN contributed to design of the study and revised the manuscript. HM drafted the manuscript. Both authors contributed to the article and approved the submitted version.

\section{FUNDING}

This study was funded by National Natural Science Foundation of China (31902232).

\section{ACKNOWLEDGMENTS}

We would like to thank Shanghai APT Biotech Co., Ltd., for assistance with the LC-MS/MS metabolomics experiments.

Niu, Y., Sun, Q., Shi, Y., Ding, Y., Li, Z., Sun, Y., et al. (2019). Immunosuppressive potential of fowl adenovirus serotype 4. Poult. Sci. 98, 3514-3522. doi: 10.3382/ ps/pez179

Niu, Y., Sun, Q., Zhang, G., Liu, X., Shang, Y., Xiao, Y., et al. (2018a). Fowl adenovirus serotype 4-induced apoptosis, autophagy, and a severe inflammatory response in liver. Vet. Microbiol. 223, 34-41. doi: 10.1016/j. vetmic.2018.07.014

Niu, Y., Sun, Q., Zhang, G., Sun, W., Liu, X., Xiao, Y., et al. (2018b). Epidemiological investigation of outbreaks of fowl adenovirus infections in commercial chickens in China. Transbound. Emerg. Dis. 65, e121-e126. doi: 10.1111/tbed.12691

Niu, Y., Sun, W., Zhang, G., Qu, Y., and Liu, S. (2016). Hydropericardium syndrome outbreak caused by fowl adenovirus serotype 4 in china in 2015. J. Gen. Virol. 97:2684. doi: 10.1099/jgv.0.00 0567

Sato, S., Solanas, G., Peixoto, F. O., Bee, L., Symeonidi, A., Schmidt, M. S., et al. (2017). Circadian reprogramming in the liver identifies metabolic pathways of aging. Cell 170, 664-677. doi: 10.1016/j.cell.2017.07.042

Schachner, A., Marek, A., Jaskulska, B., Bilic, I., and Hess, M. (2014). Recombinant FAdV-4 fiber-2 protein protects chickens against hepatitis-hydropericardium syndrome (HHS). Vaccine 32, 1086-1092. doi: 10.1016/j.vaccine.2013.12.056

Steer, P. A., Sandy, J. R., O'Rourke, D., Scott, P. C., Browning, G. F., and Noormohammadi, A. H. (2015). Chronological analysis of gross and histological lesions induced by field strains of fowl adenovirus serotypes 1 , $8 \mathrm{~b}$ and 11 in one-day-old chickens. Avian Pathol. 44, 106-113. doi: 10.1080/ 03079457.2015.1007919

Sun, H., Zhang, A. H., Yan, G. L., Piao, C. Y., Li, W. Y., Sun, C., et al. (2013). Metabolomic analysis of key regulatory metabolites in hepatitis $\mathrm{C}$ virus-infected tree shrews. Mol. Cell. Proteomics 12, 710-719. doi: 10.1074/mcp.M112.019141

Thai, M., Graham, N. A., Braas, D., Nehil, M., Komisopoulou, E., Kurdistani, S. K., et al. (2014). Adenovirus e4orf1-induced myc activation promotes host cell anabolic glucose metabolism and virus replication. Cell Metab. 19, 694-701. doi: 10.1016/j.cmet.2014.03.009

Thaker, S. K., Ch'ng, J., and Christofk, H. R. (2019b). Viral hijacking of cellular metabolism. BMC Biol. 17:59. doi: 10.1186/s12915-019-0678-9

Thaker, S. K., Chapa, T., Garcia, G. Jr., Gong, D., Schmid, E. W., Arumugaswami, V., et al. (2019a). Differential metabolic reprogramming by Zika virus promotes cell death in human versus mosquito cells. Cell Metab. 29, 1206.e12041216.e1204. doi: 10.1016/j.cmet.2019.01.024

Tohyama, S., Fujita, J., Hishiki, T., Matsuura, T., Hattori, F., Ohno, R., et al. (2016). Glutamine oxidation is indispensable for survival of human pluripotent stem cells. Cell Metab. 23, 663-674. doi: 10.1016/j.cmet.2016.03.001

Vander Heiden, M. G., Cantley, L. C., and Thompson, C. B. (2009). Understanding the warburg effect: the metabolic requirements of cell proliferation. Science 324, 1029-1033. doi: 10.1126/science.1160809 
Vastag, L., Koyuncu, E., Grady, S. L., Shenk, T. E., and Rabinowitz, J. D. (2011). Divergent effects of human cytomegalovirus and herpes simplex virus1 on cellular metabolism. PLoS Pathog. 7:e1002124. doi: 10.1371/journal.ppat. 1002124

Wang, Z., and Zhao, J. (2019). Pathogenesis of hypervirulent fowl adenovirus serotype 4: the contributions of viral and host factors. Viruses 11:741. doi: 10.3390/v11080741

Yu, Y., Maguire, T. G., and Alwine, J. C. (2011). Human cytomegalovirus activates glucose transporter 4 expression to increase glucose uptake during infection. J. Virol. 85, 1573-1580. doi: 10.1128/JVI.01967-10

Zhao, J., Zhong, Q., Zhao, Y., Hu, Y. X., and Zhang, G. Z. (2015). Pathogenicity and complete genome characterization of fowl adenoviruses isolated from chickens associated with inclusion body hepatitis and hydropericardium syndrome in China. PLoS One 10:e0133073. doi: 10.1371/journal.pone.0133073

Zhao, M., Duan, X., Wang, Y., Gao, L., Cao, H., Li, X., et al. (2020). A novel role for PX, a structural protein of fowl adenovirus serotype 4 (FAdV4), as an apoptosis-inducer in leghorn male hepatocellular cell. Viruses 12:228. doi: 10.3390/v12020228

Zhou, M., Deng, Y., Liu, M., Liao, L., Dai, X., Guo, C., et al. (2020). The pharmacological activity of berberine, a review for liver protection. Eur. J. Pharmacol. 890:173655. doi: 10.1016/j.ejphar.2020.173655
Zorova, L. D., Popkov, V. A., Plotnikov, E. Y., Silachev, D. N., Pevzner, I. B., Jankauskas, S. S., et al. (2018). Mitochondrial membrane potential. Anal Biochem. 552, 50-59. doi: 10.1016/j.ab.2017. 07.009

Conflict of Interest: The authors declare that the research was conducted in the absence of any commercial or financial relationships that could be construed as a potential conflict of interest.

Publisher's Note: All claims expressed in this article are solely those of the authors and do not necessarily represent those of their affiliated organizations, or those of the publisher, the editors and the reviewers. Any product that may be evaluated in this article, or claim that may be made by its manufacturer, is not guaranteed or endorsed by the publisher.

Copyright (c) $2022 \mathrm{Ma}$ and Niu. This is an open-access article distributed under the terms of the Creative Commons Attribution License (CC BY). The use, distribution or reproduction in other forums is permitted, provided the original author(s) and the copyright owner(s) are credited and that the original publication in this journal is cited, in accordance with accepted academic practice. No use, distribution or reproduction is permitted which does not comply with these terms. 